\title{
Ultra-flat coplanar electrodes for controlled electrical contact of molecular films
}

\author{
Florent Martin, ${ }^{1,2}$ Bas Hendriksen, ${ }^{1, a)}$ Allard Katan, ${ }^{1}$ Imma Ratera, ${ }^{1, b)}$ Yabing Qi, ${ }^{1,3, c)}$ \\ Bruce Harteneck, ${ }^{3}$ J. Alexander Liddle, ${ }^{3, d)}$ and Miquel Salmeron ${ }^{1,2,3}$ \\ ${ }^{1}$ Materials Science Division, Lawrence Berkeley National Laboratory, Berkeley, California 94720, USA \\ ${ }^{2}$ Materials Science and Engineering Department, University of California at Berkeley, California 94720 , \\ USA and Applied Science and Technology Graduate Group, University of California, Berkeley, \\ California 94720, USA \\ ${ }^{3}$ The Molecular Foundry, Lawrence Berkeley National Laboratory, Berkeley, California 94720, USA
}

(Received 20 September 2011; accepted 6 November 2011; published online 14 December 2011)

\begin{abstract}
Reliable measurement of electrical charge transport in molecular layers is a delicate task that requires establishing contacts with electrodes without perturbing the molecular structure of the film. We show how this can be achieved by means of novel device consisting of ultra-flat electrodes separated by insulating material to support the molecular film. We show the fabrication process of these electrodes using a replica technique where gold electrodes are embedded in a silicon oxide film deposited on the angstrom-level flat surface of a silicon wafer. Importantly, the co-planarity of the electrode and oxide areas of the substrate was in the sub-nanometer range. We illustrate the capabilities of the system by mapping the distribution of electrical transport pathways in molecular thin films of self-assembled oligothiophene derivatives using conductive atomic force microscopy. In comparison with traditional bottom contact non-coplanar electrodes, the films deposited on our electrodes exhibited contact resistances lower by a factor of 40 than that of the similar but non-coplanar electrodes. (c) 2011 American Institute of Physics. [doi:10.1063/1.3664789]
\end{abstract}

\section{INTRODUCTION}

A molecular level understanding of the structural and electrical properties of organic thin films provide the basis for the optimization of their structure for applications in photovoltaics, switches and sensors. ${ }^{1}$ To correlate molecular structure and charge transport properties, bulk and surface characterization techniques have been extensively employed. For example, X-ray and electron diffraction provide the symmetry and size of the unit cell and the orientation of crystalline domains. ${ }^{2}$ Scanning probe microscopy (SPM) makes possible to characterize film morphology, molecular lattice structure, and defects such as grain boundaries. The degree of crystallinity and the density of defects are key parameters that influence carrier mobility in organic materials. However, combining electrical and structural characterization of molecularly thin organic films is challenging because of the difficulty of contacting the fragile organic materials without causing uncontrolled structural changes or damage. ${ }^{3}$ Our ultra-flat coplanar electrodes make also possible to examine the detailed structure of the contact between organic films and electrodes, which is crucial in determining the contact resistance and intrinsic electrical properties in short channel devices.

Various techniques have been employed to provide noninvasive electrical contacts with thin films. Boggild et al. designed multi actuated probes for 4-point measurement on

a) Present address: Institute for Molecules and Materials, Radboud University, 6525 AJ Nijmegen, The Netherlands.

b) Present address: Institut de Ciencia de Materials de Barcelona, Spain.

c) Present address: Department of Electrical Engineering, Princeton University, Princeton, New Jersey 08544, USA.

d) Present address: National Institute of Standards, Gaithersburg, Maryland 20899, USA. ultra-thin organic films and combined this setup with scanning Kelvin probe force microscopy (SKFM) ${ }^{4}$ Smit et al. studied monolayer organic field-effect transistors (FET) fabricated by under-etching gold electrodes separating a $\mathrm{SiO}_{2}$ dielectric covered by self-assembled monolayer (SAM) of silanes that extended underneath the electrodes. ${ }^{5}$ In another study, Cao et al. ${ }^{6,7}$ used graphene to establish electrical contact with the organic layers.

An alternative method to create non-perturbative contacts is to embed metal electrodes in the surface of an insulator to create a flat, co-planar metal-insulator-metal substrate over which an organic film can be deposited to form the channel material of an FET. ${ }^{8,9}$ An atomically smooth transition between the metal electrodes and the insulator surface would help minimize structural modifications of the organic film at the contact. In addition, the planar configuration would make it possible to study the structure of the organic film by high resolution AFM. However, fabrication of embedded electrodes with planarity and flatness at the molecular scale is very challenging. In several studies, Xu et al. ${ }^{10,11}$ created embedded electrodes by first etching a silicon oxide substrate patterned by UV lithography followed by the evaporation of a layer of gold with a thickness equal to the etched material. However, the authors observed that the etch process produced trenches between the electrode and the dielectric.

Using a similar method, Singh et al. obtained also a planar surface with electrodes decorated by a trench $50 \mathrm{~nm}$ wide and $5 \mathrm{~nm}$ deep. ${ }^{12}$ Higuchi et al. added mechanical polishing to the protocol in order to smooth the device. ${ }^{13} \mathrm{Xu}$ and Singh compared the performance of their planar transistors with regular bottom contact transistors and reported charge mobility $30 \%-70 \%$ higher, while the contact resistance and 
threshold voltage were up to $50 \%$ lower in the planar configuration. Xu studied $60 \mathrm{~nm}$ thick films of pentacene and P3HT while Singh focused on P3HT nano-fibrils 50-100 nm thick. If similar electrodes are to be used with much thinner materials such as organic monolayers, the co-planarity requirements are likely to be much more stringent.

Another method to fabricate embedded coplanar electrodes is based on a replica technique, as demonstrated by Tsutsui et al. ${ }^{14}$ In that approach electrodes are first fabricated on top of a flat sacrificial support, followed by deposition of an insulating dielectric layer. The top of the insulator layer is then bonded to a second support substrate. Finally, the sacrificial substrate is removed by chemical-mechanical polishing. Using this technique, they obtained nearly coplanar devices with less than $1 \mathrm{~nm}$ peak to peak roughness.

In this paper, we present results using a substantially improved replica technique that produces embedded microelectrodes with a planar configuration free of trenches at the electrode edges. We tested our electrodes by studying monolayers of semiconductor molecules. The results were then compared with those obtained with conventional bottomcontact electrodes. We show that crystalline monolayer islands can be contacted electrically and that a 40 -fold reduction in the contact resistance can be obtained compared to conventional $20 \mathrm{~nm}$ thick electrodes overlaid on the silicon oxide wafer. We also show that electrodes separated by less than $100 \mathrm{~nm}$ can also be fabricated with electron-beam lithography, demonstrating that the replica method is also adequate to create coplanar nano-electrodes.

\section{EXPERIMENTAL}

\section{A. Patterning of silicon wafers with Au electrodes}

The roughness of the electrode devices produced by the replica method is determined by the roughness of the sacrificial substrate. We used standard 6" polished silicon $\langle 100\rangle$ wafers with $0.2 \mathrm{~nm}$ RMS roughness as the sacrificial substrate. A pattern containing 2-5 and 4-terminal electrodes was defined with standard UV or e-beam lithography using FujiFilm OiR 700-10 or PMMA resist, respectively. The gap between the UV lithographically defined electrodes at the center of the 4-electrode set was chosen to be between $2 \mu \mathrm{m}$ and $500 \mathrm{~nm}$, which is the resolution limit of the stepper used in our set up (GCA 8500). The gaps of the electron beam patterned electrodes were below $100 \mathrm{~nm}$ and the electrode widths were $200 \mathrm{~nm}$. The electrodes were deposited using an e-beam evaporator and had nominal thickness between $12 \mathrm{~nm}$ and $15 \mathrm{~nm}$. No adhesion layer was used in order to achieve minimal roughness over the wafer. Adhesion layers such as titanium or chromium layers have RMS roughness two to three times higher than a polished silicon wafer with its native oxide, as measured by AFM. The wetting properties of gold on $\mathrm{SiO}_{2}$ imposed a minimum Au thickness of $12 \mathrm{~nm}$ in order to obtain a continuous metallic film. Lower thicknesses led to a network of poorly connected gold grains. After gold evaporation the resist lift-off was performed by soaking the wafer for $30 \mathrm{~min}$ in PRS- 1000 (Baker) at $75^{\circ} \mathrm{C}$ for the UV patterned structures and by soaking in dichloromethane at room temperature for the e-beam patterns [Fig. 1(a)].

\section{B. Oxide deposition and bonding to borofloat glass}

In the next step [Fig. 1(b)], a silicon oxide layer was deposited with a nominal thickness of $150 \mathrm{~nm}$ on the gold patterned wafer by Plasma Enhanced Chemical Vapor Deposition (PECVD) of a silane $\left(\mathrm{SiH}_{4}\right)$ precursor with nitrous oxide $\left(\mathrm{N}_{2} \mathrm{O}\right)$ at $350^{\circ} \mathrm{C}$. The PECVD oxide covered the gold patterned surface in a conformal manner. The height variations due to the presence of the electrodes were preserved in the topography of the oxide film after oxide deposition. Next a $6^{\prime \prime}$ borofloat glass wafer was bonded to the wafer on the oxide side by anodic bonding at $300{ }^{\circ} \mathrm{C}$ [Fig. 1(c)]. The electrode was biased to $-1000 \mathrm{~V}$ on the silicon wafer side while the electrode on the glass side was grounded.

\section{Dicing, polishing, and etching}

The $\mathrm{Si}$-wafer/ $\mathrm{Au} / \mathrm{SiO}_{2} /$ Boroglass stack was diced in pieces of $1 \mathrm{~cm}^{2}$ in area by cleaving along the crystallographic directions of silicon using a diamond scribe. A Logitech lapping machine was used to polish the silicon wafer side until $\sim 50 \mu \mathrm{m}$ of the interface [Fig. 1(d)]. The remaining $50 \mu \mathrm{m}$ were subsequently etched in an aqueous solution of $25 \%$ tetramethylammonium hydroxide (TMAH) [Fig. 1(e)]. The etching was performed initially at $70{ }^{\circ} \mathrm{C}$ at a rate of $1-2 \mu \mathrm{m} / \mathrm{min}$. We observed that the insulator part of the exposed electrode pattern device had a higher than desired RMS roughness after etching of the silicon oxide with the hot TMAH, which we attribute to the limited etching selectivity of $\mathrm{Si}$ vs $\mathrm{SiO}_{2}$ at high temperature. ${ }^{15}$ Due to inhomogeneity of the etching, exposure of the oxide to TMAH could not be avoided while still etching away all the silicon. For this reason the final few microns of the Si wafer were etched at room temperature. This also helped minimize damage to the gold electrodes. A disadvantage of the room temperature etching is its slowness, and typically took several hours. After etching, the samples were rinsed with MilliQ water to remove traces of TMAH.

\section{Atomic force microscopy and monolayer preparation}

The coplanar electrode structures were characterized using AFM in contact and tapping modes with Agilent 5500 and Veeco Icon instruments. For roughness measurements, $0.2 \mathrm{~N} / \mathrm{m}$ silicon tips from Nanoworld were used. The RMS roughness of the electrodes and oxide was measured using the open source SPM image analysis program Gwyddion. To test the quality of the coplanar electrode device and their ability to establish electrical contact with molecular layers we deposited molecularly films of D5TBA, an oligothiophene on the electrodes using the Langmuir-Blodgett technique. The molecules were first dissolved in chloroform. A droplet of the solution was then deposited at the air-water interface in a Langmuir-Blodgett trough. The molecules self-assembled 

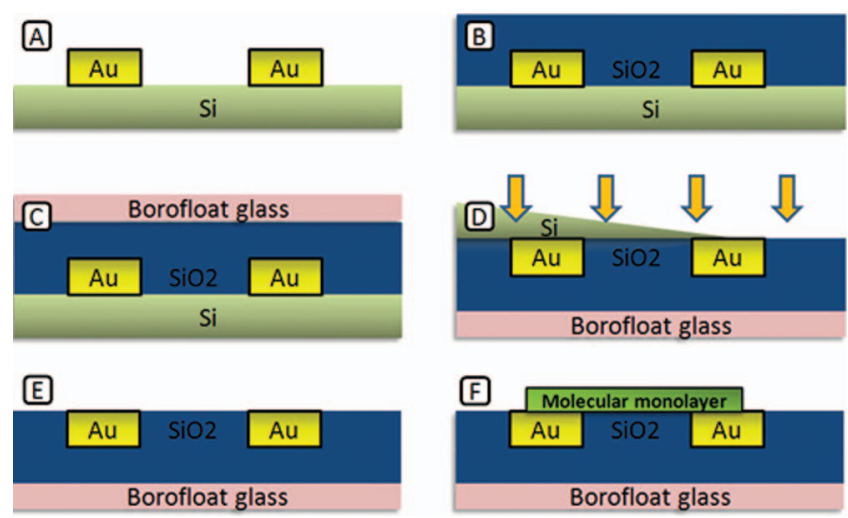

FIG. 1. (Color online) Fabrication of ultra-flat coplanar electrodes. (a) Patterning and evaporation of $20 \mathrm{~nm}$ thick Au electrodes. (b) PECVD of $150 \mathrm{~nm}$ of silicon oxide. (c) Anodic bonding to a glass wafer. (d) Mechanical polishing followed by etching in TMAH at $70^{\circ} \mathrm{C}$. (e) The final few microns are etched in TMAH 25\% at room temperature. (f) Organic layer deposition using the LB technique.

into polycrystalline islands which diameters varying between $500 \mathrm{~nm}$ and $2 \mu \mathrm{m}$, which were subsequently transferred to the coplanar electrodes [Fig. 1(f)].

\section{RESULTS AND DISCUSSION}

\section{A. Characterization of the electrodes}

The ultra-flat electrodes were characterized by AFM to determine the roughness and coplanarity of the dielectric/gold interface. Figure 2(a) shows a tapping mode AFM image of a region containing four embedded coplanar gold electrodes. Long range undulations of approximately $10 \mathrm{~nm}$ amplitude can be observed around the electrodes (Fig. 2(b)). These height variations are due to the $12 \mathrm{~nm}$ gold electrode thickness, which is conformally reproduced by the PECVD oxide deposited on the patterned wafer. The interfacial stress relaxes when the silicon layer is etched away and produces the observed smooth long range undulations. Since these variations are smooth over several micrometers they are not expected to induce perturbations in the molecular scale structure of the deposited films.

RMS roughness in the silicon oxide dielectric region was found to be $0.3 \mathrm{~nm}$, similar to that of the thermal oxides used as dielectrics in organic FETs (0.4-0.5 nm RMS). It is also sufficiently low to ensure preservation of the molecular alignment in the fragile organic film bridging the gap between electrodes. In contrast, the roughness in the gold electrode regions was about $1 \mathrm{~nm}$, as shown in the height profile in fig. 2(b). This is a result of the grainy character of the gold film that poorly wets the $\mathrm{SiO}_{2}$. The expanded AFM image in fig. 2(c) shows gold grains with sizes in the order of $100 \mathrm{~nm}$. The resistance of the gold film was below $100 \Omega$, indicating that the grains are joined to form a continuous conductive film.

In addition to the roughness of the oxide and gold surfaces, the degree of co-planarity, i.e., the step at the transition between the gold electrodes and the dielectric is of the upmost importance and should be as small as possible. The arrows in Figs. 2(a) and 2(b) indicate the location of the $\mathrm{Au} / \mathrm{SiO}_{2}$ interface. The step heights were $1 \mathrm{~nm}$ and $0.3 \mathrm{~nm}$, respectively, for
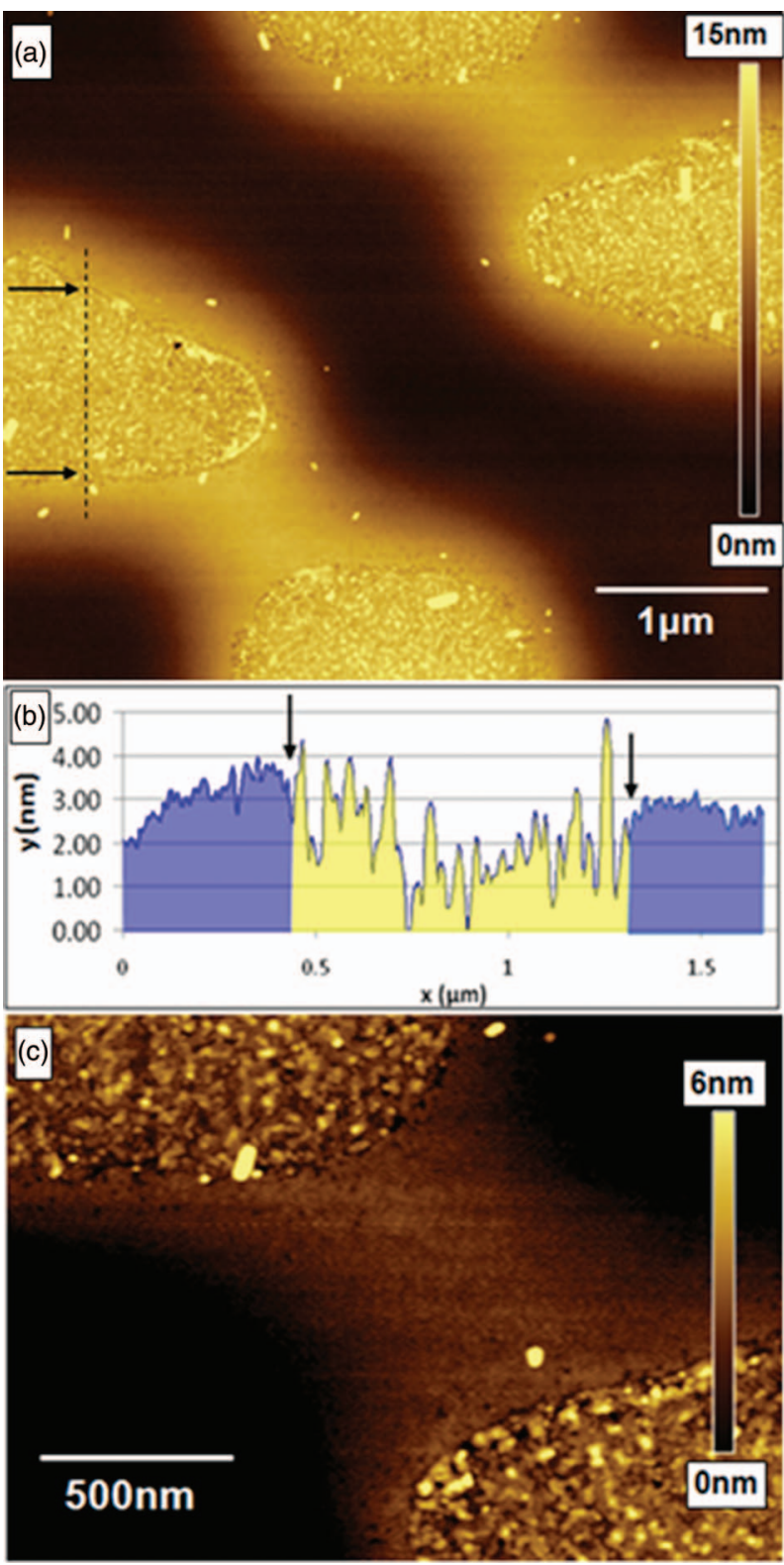

FIG. 2. (Color online) (a) Topographic AFM image showing 4 gold electrodes embedded in silicon oxide. (b) Height profile along the black dotted line across the electrode on the left. The $\mathrm{Au} / \mathrm{SiO}_{2}$ boundaries are indicated by arrows. The top boundary has a step $1 \mathrm{~nm}$ high and the bottom one $0.3 \mathrm{~nm}$. (c) Expanded view of the gap between the two top right electrodes.

the top and bottom interfaces at the indicated positions. On average the steps height was found to be $0.5 \mathrm{~nm}$, with lower values found often (but randomly) in some of the electrodes. The step at the interface is primarily due to the transition from smooth oxide to the rougher grainy gold surface.

\section{B. AFM characterization of the organic monolayers}

We used the Langmuir-Blodgett (LB) technique to deposit a monolayer film of decylpentathiophene butyric acid (D5TBA) molecules on the embedded electrode substrates. These oligothiophene molecules self-assemble into $3 \mathrm{~nm}$ thick flower-like islands at the air-water interface of the Langmuir trough. ${ }^{16}$ They consist of single crystal domains 

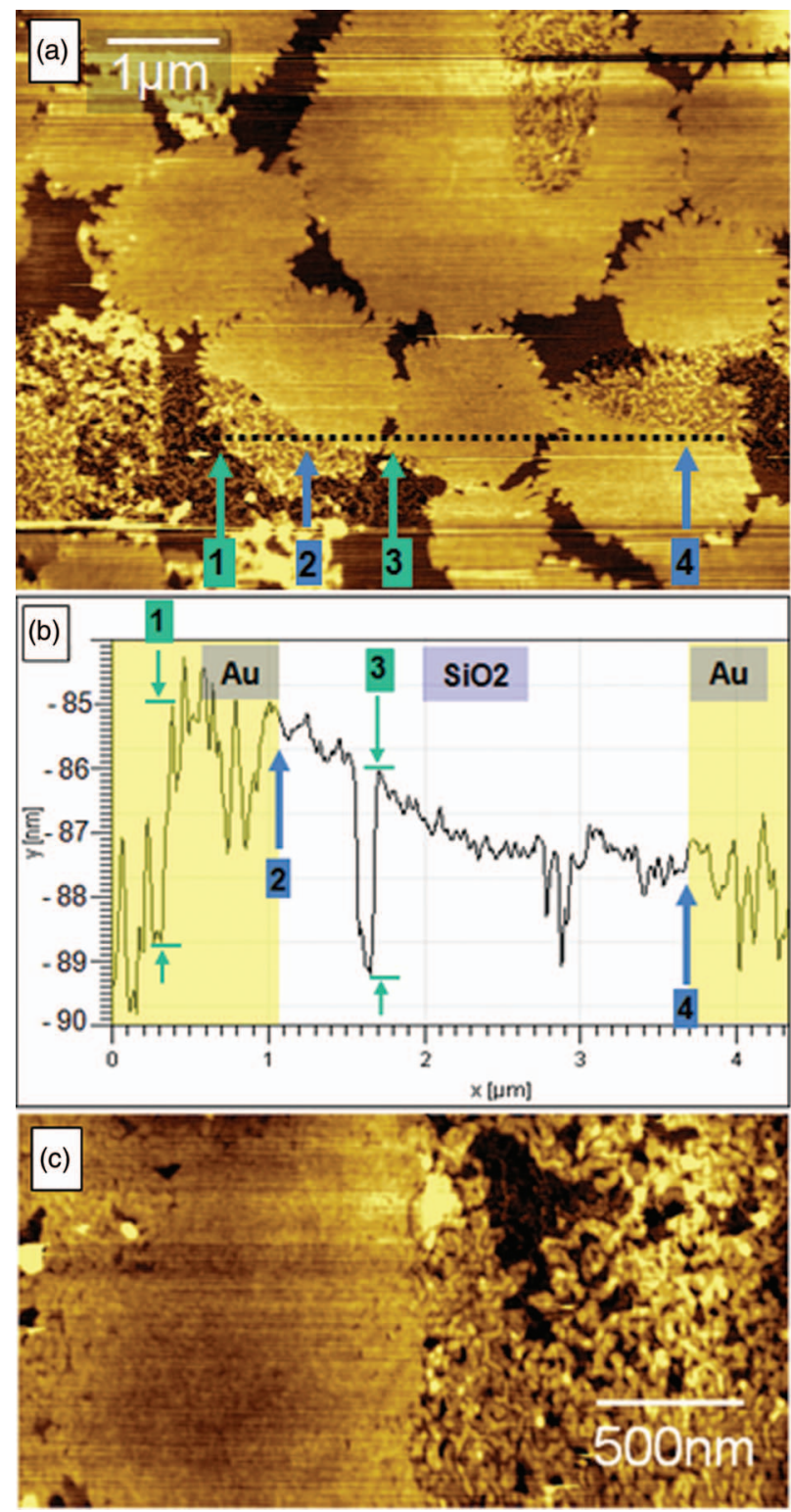

FIG. 3. (Color online) (a) Topographic AFM image of 3 gold electrodes embedded in silicon oxide. (b) Height profile along the black dotted line. Numbers 2 and 4 mark the $\mathrm{Au} / \mathrm{SiO}_{2}$ transition, with steps less than $0.5 \mathrm{~nm}$ high. Positions 1 and 3 mark the edges of two molecular islands, with a height of $2.6 \mathrm{~nm}$. (c) Expanded view of a D5TBA island overlapping the $\mathrm{SiO}_{2} / \mathrm{Au}$ interface showing that the monolayer adapts conformally to the grainy structure of the Au electrode.

separated by boundaries oriented roughly radially from the center. We found that the crystalline structure and morphology of the monolayer film was the same regardless of the substrate where it was deposited, including $\mathrm{Au}, \mathrm{SiO}_{2}$, and mica. The film consists of islands with diameters in the range of $500 \mathrm{~nm}$ to $2 \mu \mathrm{m}$, as shown in Fig. 3, with many of them overlapping the electrodes. A height profile along the dashed line in Fig. 3(a) is shown in Fig. 3(b), with the edge of the electrodes indicated by blue arrows. The step height between electrode and dielectric is a few tenths of a nanometer at both $\mathrm{Au} / \mathrm{SiO}_{2}$ interfaces. The locations indicated by arrows 1 and 3 correspond to discrete $2.6 \mathrm{~nm}$ steps at the edges of molecular islands. The RMS roughness on the gold electrodes and the oxide are $1 \mathrm{~nm}$ and $0.3 \mathrm{~nm}$, respectively, as indicated earlier.

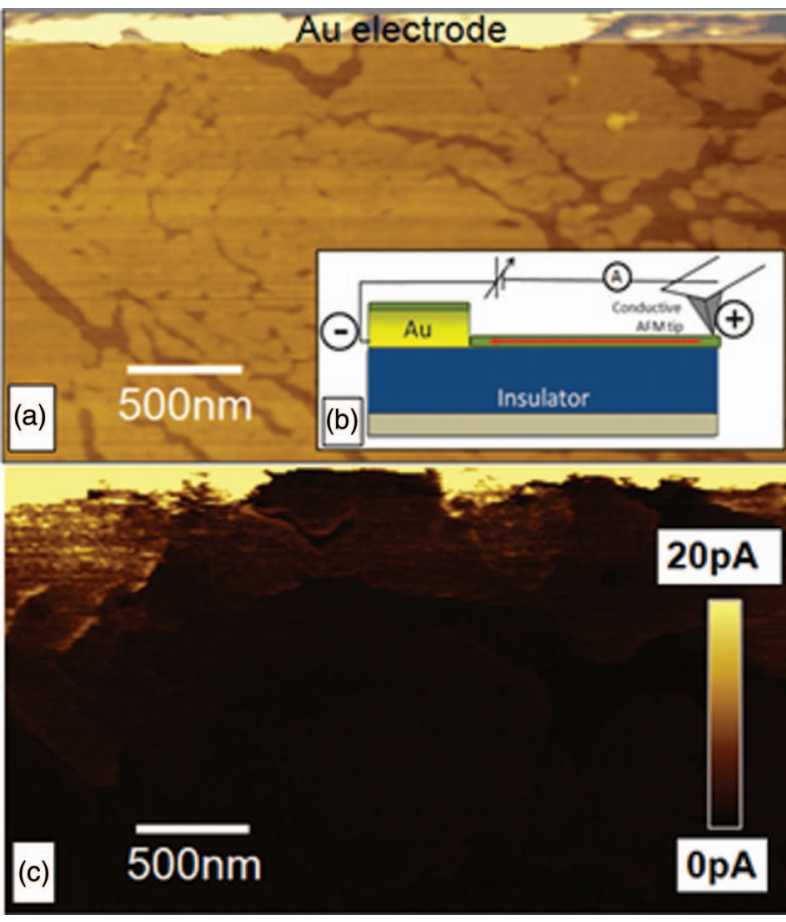

FIG. 4. (Color online) (a) AFM topographic image of a D5TBA organic crystalline film deposited on a silicon oxide wafer patterned with $20 \mathrm{~nm}$ thick gold electrodes. (b) Schematic of the setup used to probe the conductivity of the monolayer film and its contact resistance with the gold electrode. (c) Current image showing the current from the tip and the electrode as a function of position of the contact. Several monolayer islands are electrically connected to the gold electrode located at the top of the image.

\section{Electrical contact between organic layers and electrodes}

The electrical contact between organic monolayer and electrodes was investigated by measuring the current flowing through the D5TBA monolayer islands from the AFM tip in contact with the molecular island to one of the embedded electrodes. We used normal loads of 5-10 nN, which we found to be sufficiently low to ensure that no deformation of the molecular film occurs. ${ }^{16}$ The measured current did not vary significantly with applied load in this regime. To compare with the commonly used non-coplanar electrode devices we patterned similarly shaped $20 \mathrm{~nm}$ thick electrodes onto silicon substrates with $100 \mathrm{~nm}$ of thermally grown oxide. A schematic of the experiments on the non-coplanar and the co-planar electrodes is shown in the insets of fig. 4(b) and fig. 5(b). In both cases the AFM tip was grounded while a $-5 \mathrm{~V}$ bias voltage was applied to the gold electrode via a contact made by wire bonding.

Figs. 4(a) and 4(c) show topography and current images of a monolayer located close to the edge of the non-coplanar gold electrode located at the top of the image (bright yellow region). The current image shows that most of the islands in physical contact with the electrode were also electrically connected. When the tip contacted the film at less than $100 \mathrm{~nm}$ distance from the electrode the current varied between $20 \mathrm{pA}$ and $50 \mathrm{pA}$, which corresponds to a total series resistance of $100-250 \mathrm{G} \Omega$ at $-5 \mathrm{~V}$. This resistance is the sum of 3 terms: the tip-film contact resistance, the intrinsic resistance of the 

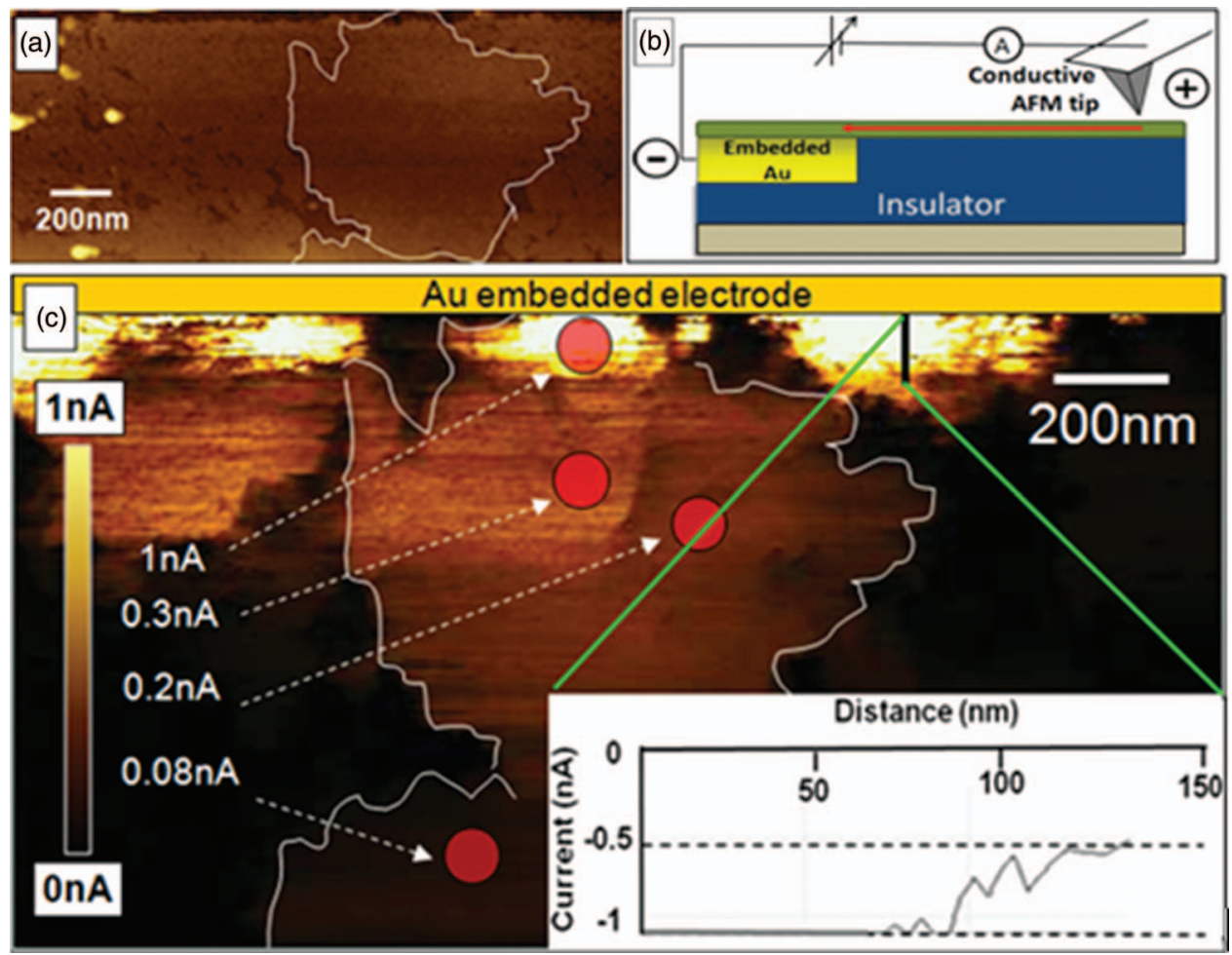

FIG. 5. (Color online) (a) AFM topographic image of a D5TBA film deposited on the ultra-flat electrodes. The white contour marks the boundary of a particular island of D5TBA. (b) Schematic of the set up to probe the conductivity of the monolayer and the contact resistance between the monolayer and embedded electrode. (c) Current image showing that some monolayer islands are electrically well connected to the gold electrode located at the top of the image. The central island, emphasized by a white contour, corresponds to the island shown in (a). The color contrast corresponds to the current level (vertical bar) in various parts of the islands. Profile along the black line is shown in the inset.

film, and the contact resistance at the electrode. An approximate value for the tip-film contact resistance can be obtained by measuring the current when the tip is in contact with the monolayer at a position on top of the Au electrode. The current there was larger than $1 \mathrm{nA}$ implying that the tip-film contact resistance was smaller than $5 \mathrm{G} \Omega$. In addition, the resistance of the molecular film is in the range 1-20 G $\Omega$ as shown by the results of CAFM on embedded electrodes in fig. 5 described below. The total resistance therefore is dominated by the contact resistance at the electrode-film interface. Although in principle the conductivity of the film and the resistance of the grain boundaries could be derived from spatially resolved current measurements with the AFM tip, the low level of the current which was close to the noise level of the instrument (a few $\mathrm{pA}$ ) prevented accurate determination of these parameters. In addition, in some cases molecular islands that appeared to be in physical contact with the electrode did not show any current, implying that they are not electrically connected or had contact resistances in the hundreds of G $\Omega$. These islands were probably disordered or otherwise damaged at the electrode interface. We will now show that the planar electrodes configuration is more suitable for determining properties of the film due to the lower contact resistance with the gold electrode.

Figs. 5(a) and 5(c) show topography and current images of the D5TBA monolayer film at the edge of an embedded, coplanar gold electrode. Note that the current scale in Fig. 5(c) is 50 times larger than in Fig. 4(c). The current varied from tens of $\mathrm{pA}$ to $\geq 1 \mathrm{nA}$ (saturation of the current amplifier) close to the electrode edge, which corresponds to a total resistance from the AFM tip through the monolayer to the electrode between $1 \mathrm{G} \Omega$ and $20 \mathrm{G} \Omega$. The current profile in the inset shows a sharp gradient $(0.5 \mathrm{nA}$ in $50 \mathrm{~nm})$, with the current reaching saturation of the current amplifier when the tip was within $100 \mathrm{~nm}$ of the electrode. Extrapolation of the current to zero tip-electrode distance gives a current higher than $2 \mathrm{nA}$. Correspondingly we estimate the sum of the tip-film and filmelectrode contact resistances to be below $2.5 \mathrm{G} \Omega$.

The details of the current image show that for D5TBA islands overlapping with the electrodes the current varies within a single island. This implies the electrical circuit is not dominated by the gold-island contact resistance, but is significantly modulated by the conductance of the islands themselves. Close to the electrodes the current is saturated, dropping to $0.3 \mathrm{nA}$ in the top left region of the molecular islands, which corresponds to an increase in resistance in the order of $10 \mathrm{G} \Omega$. In the top right-hand region of the central island at the same distance from the electrode the current is only $200 \mathrm{pA}$ and there is a sharp step in the current between these two regions. The fast scan direction of the AFM tip is horizontal, so that the appearance of a sharp vertical boundary cannot be attributed to a change in the state of the AFM tip. Our studies of D5TBA islands inform us that this corresponds to a grain boundary, the reduction in current indicating a grain boundary resistance on the order of $10 \mathrm{G} \Omega$. Finally, a current of $80 \mathrm{pA}$ is measured on the bottom island, which is in physical contact with the large central island through an inter-island boundary. Kelley et al. measured individual grain 
boundary resistance in sexithiophene monolayers connected to a gold nanowire with a conducting AFM tip and found a resistance in the order of $25 \mathrm{G} \Omega .{ }^{17}$ Using a doped $\mathrm{Si}$ gate, they also found that the GB resistance could be modulated by an applied bias. ${ }^{18}$ The value obtained by Kelley et al. is close to the $10 \mathrm{G} \Omega$ measured in this study.

From these results we can reach two conclusions. For non-planar electrodes ( $20 \mathrm{~nm}$ thick in our case), where the contact resistance varies between $100 \mathrm{G} \Omega$ and open circuit, little information can be obtained about the conductivity of the organic monolayers because the contact resistance masks any features specific to islands. For embedded electrodes, the contact resistance is below the resistance of the crystalline monolayer and grain boundaries. The resistance of a grain boundary in D5TBA is close to $10 \mathrm{G} \Omega$.

\section{Nano-electrodes by e-beam lithography}

AFM images of coplanar electrodes made by e-beam lithography are shown in Fig. 6. We fabricated electrodes

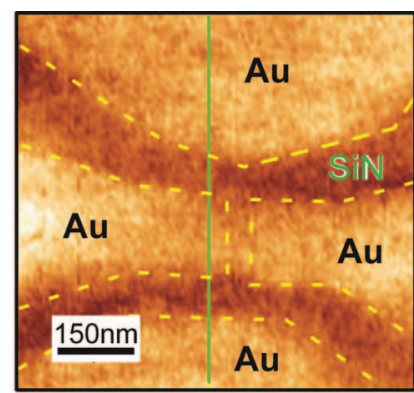

(a)

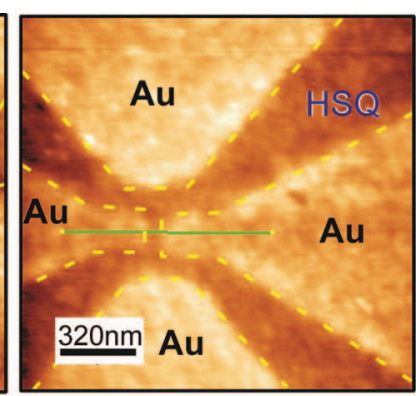

(b)
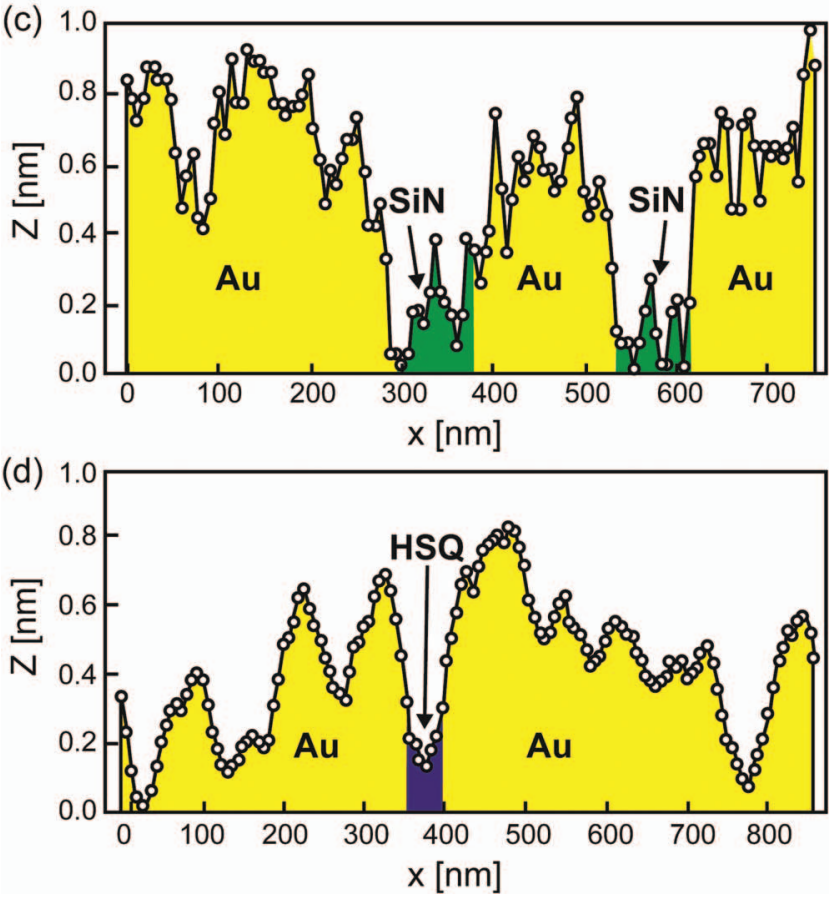

FIG. 6. (Color online) AFM images of co-planar nano-electrodes fabricated by the replica process and e-beam lithography using SiN (a) and HSQ dielectric material (b). The dashed lines mark the edges of the electrodes. (c) and (d) Height profiles across electrodes along the lines in (a) and (b). In all cases the electrode-dielectric step is below $1 \mathrm{~nm}$. with SiN and HSQ as embedding insulators. The SiN was deposited by PECVD and the HSQ is a curable spin-on insulator material. The SiN containing electrodes were produced in the same way as those fabricated using UV lithography. Electrode structures, containing HSQ as an insulator, were fabricated using an ultra-flat nickel etch stop layer, which was first deposited on the silicon sacrificial substrate before electrode patterning and deposition. Similar to the UV-defined electrodes we observed long range smooth height variations due to film relaxation. This effect was significantly reduced in the gaps narrower than $100 \mathrm{~nm}$, resulting in smaller height variation at the transition between the gold and the nitride or oxide. The co-planarity at the electrode gap was within $0.5 \mathrm{~nm}$. The roughness on the nitride and oxide had a comparable value to the oxide of UV-defined electrodes and the roughness on the gold was, in contrast to the UV electrodes, well below $0.4 \mathrm{~nm}$. These results demonstrate that the replica method is also applicable for creating nano-size, coplanar electrode structures.

\section{CONCLUSIONS}

We have developed a method for fabricating electrodes embedded in insulators (silicon oxide and nitride) that are coplanar within a fraction of a nanometer. The roughness of the dielectric and electrode areas was $0.3 \mathrm{~nm}$ and $1 \mathrm{~nm}$ RMS, respectively. Using an oligothiophene derivative (D5TBA) prepared by the Langmuir-Blodgett technique we demonstrated that these ultra-flat electrode devices are suitable for measurements of the electrical properties of organic films of nanometer thickness. In comparison with the $20 \mathrm{~nm}$ thick deposited gold electrodes the electrode-molecular film resistance decreased more than 40 -fold. From our test case study we can conclude that electrical conduction through 2D crystals of D5TBA is grain boundary dominated.

\section{ACKNOWLEDGMENTS}

We acknowledge Jeff Beeman and Ed Wong for technical support, and the CXRO at LBNL for the use of sputter deposition equipment. AFM measurements and fabrication of e-beam lithography were performed at the Molecular Foundry. Fabrication of optical lithography patterned devices was performed at the UC Berkeley Microlab. This work was supported by the Office of Science, Office of Basic Energy Sciences, of the U.S. Department of Energy under Contract No. DE-AC02-05CH11231. Imma Ratera acknowledges a grant from the Generalitat de Catalunya Nanotech fellowship program.

The full description of the procedures used in this paper requires the identification of certain commercial products and their suppliers. The inclusion of such information should in no way be construed as indicating that such products or suppliers are endorsed by NIST or are recommended by NIST or that they are necessarily the best materials, instruments, or suppliers for the purposes described.

\footnotetext{
${ }^{1}$ S. R. Forrest, Nature (London) 428, 911 (2004).

${ }^{2}$ M. L. Chabinyc, Polym. Rev. 48, 463 (2008).
} 
${ }^{3}$ E. A. Weiss, J. K. Kriebel, M. A. Rampi, and G. M. Whitesides, Phil. Trans R. Soc. A 365, 1509 (2007)

${ }^{4}$ P. Bøggild, F. Grey, T. Hassenkam, D. R. Greve, and T. Bjørnholm, Adv. Mater. 12, 947 (2000).

${ }^{5}$ E. C. P. Smits, S. G. J. Mathijssen, P. A. van Hal, Sepas Setayesh, T. C. T. Geuns, K. A. H. A. Mutsaers, E. Cantatore, H. J. Wondergem, O. Werzer, R. Resel, M. Kemerink, S. Kirchmeyer, A. M. Muzafarov, S. A. Ponomarenko, B. D. Boer, P. W. M. Blom, and D. M. de Leeuw, Nature (London) 455, 2 (2008).

${ }^{6}$ Y. Cao, S. Liu, Q. Shen, K. Yan, P. Li, J. Xu, D. Yu, M. L. Steigerwald, C. Nuckolls, Z. Liu, and X. Guo, Adv. Funct. Mater. 19, 2743 (2009).

${ }^{7}$ Y. Cao, Z. Wei, S. Liu, L. Gan, X. Guo, W. Xu, M. L. Steigerwald, Z. Liu, and D. Zhu, Angew. Chem. 122, 6463 (2010).

${ }^{8}$ Y. Luo, F. Gustavo, J.-Y. Henry, F. Mathevet, F. Lefloch, M. Sanquer, P. Rannou, and B. Grévin, Adv. Mater. 19, 2267 (2007).

${ }^{9}$ S. Johnson, D. Evans, A. G. Davies, E. H. Linfield, and C. Walti, Nanotechnology 20, (2009).
${ }^{10}$ M. S. Xu, M. Nakamura, M. Sakai, and K. Kudo, Adv. Mater. 19, 371 (2007).

${ }^{11}$ M. Xu, K. Nagai, M. Nakamura, K. Kudo, and M. lizuka, Appl. Phys. Lett., 90, $223512(2007)$

${ }^{12}$ K. A. Singh, T. Young, R. D. McCullough, T. Kowalewski, and L. M. Porter, Adv. Funct. Mater. 20, 2216 (2010).

${ }^{13}$ Y. Higuchi, N. Ohgami, M. Akai-Kasaya, A. Saito, M. Aono, and Y. Kuwahara, Jpn. J. Appl. Phys. 45, 145 (2006).

${ }^{14} \mathrm{~K}$ Tsutsui, M Nakata, M Morita, M Tokuda, K Nagatsuma, H Onozato, T Kaneko, T Edura, Y Mita, and H Koinuma, Curr. Appl. Phys. 7, 329 (2007).

${ }^{15}$ K. Biswas and S. Kal, Microelectron. J. 37, 519 (2006)

${ }^{16}$ B. L. M. Hendriksen, F. Martin, Y. Qi, C. Mauldin, N. Vukmirovic, J. F. Ren, H. Wormeester, A. J. Katan, V. Altoe, S. Aloni, J. M. J. Fréchet, L. W. Wang, and M. Salmeron, Nano Lett. 10, 4107 (2011).

${ }^{17}$ T. W. Kelley, E. Granstrom, and C. D. Frisbie, Adv. Mater. 11, 261 (1999).

${ }^{18}$ T. W. Kelley and C. D. Frisbie, J. Phys. Chem. B 105, 4538 (2001). 